\title{
The Fluid Dynamics of Rising and Evaporating Droplet in an Immiscible Liquid
}

\author{
Abdullah Abbas Kendoush \\ Department of Nuclear Engineering Technology \\ Augusta Technical College, Augusta, Ga, USA \\ akendoush@augustatech.edu
}

\begin{abstract}
Analytical solutions were obtained for the virtual mass coefficient, drag coefficient and the equation of motion of an evaporating drop and a growing bubble within the drop that rises in an immiscible liquid. The derived theoretical results were agreeable with the available experimental data.
\end{abstract}

Keywords: Direct-contact heat transfer, Bubbles, Evaporating drop, Virtual mass coefficient, Drag coefficient.

\section{Introduction}

Direct-contact heat transfer between a drop rising and vaporizing in a continuous, stagnant and immiscible liquid whose temperature is above the boiling point of the drop has extensive applicability in a number of engineering fields. One of the important application is the process of desalination of sea or brackish water by freezing. The phenomenon is complicated due to continuous evaporation of the drop during its rise. This makes its size changing with time as shown in Fig.1. Since the velocity of rise of bubble or drop is a strong function of size and the size is expanding, hence the velocity becomes a function of time.

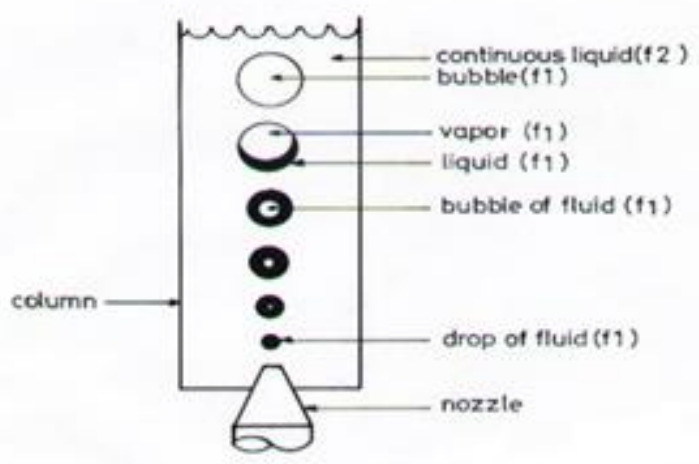

Fig. 1 Schematic sketch of an evaporating drop in an immiscible liquid

Simpson et al. [1] showed experimentally that the instantaneous velocity of the evaporating butane droplet was constant at about $0.25 \mathrm{~m} / \mathrm{s}$ up to a diameter ratio of 2.0 in brine and 2.7 in water. Thereafter, the velocity varied as the square root of the diameter ratio. This ratio is between the instantaneous diameter and the inlet drop diameter.

Selecki and Gradon [2] transformed the equation of motion into a Ricatti type and solved it numerically to obtain the travel distance of the drop as a function of time. They used $1 / 2$ as the value of the virtual mass coefficient in their solution which was agreeable with their experimental data.

The only analytical solution, as far as another is aware, is that of Raina and Wanchoo [3] who derived an equation for the velocity of rise of the drop, simply, by equating the drag to buoyancy. This equation is not easy to use due to its complex type. The following facts were not taken into account during the derivation:

(i) The buoyancy due to the vapor within the drop.

(ii) The virtual mass of the two-phase drop. 
(iii) The transient motion of the drop.

The pioneer works of Shimaoka and Mori [4] produced a correlation for the instantaneous velocity of the liquid/vapor two-phase bubble. The finite element method in Langrangian from was applied by Dammel and Beer [5] to solve the Navier-Stokes equations. They found a vortex generated within the liquid of the drop as it rises up inside the immiscible liquid.

The aim of the present work is to get more understanding of the hydrodynamics associated with the evaporation droplet during its rise with heat absorption from the continuous phase. This will be achieved through the analytical solution of the equation of motion by considering the virtual mass effect and the interfacial drag forces.

\section{2. Theoretical analysis}

\subsection{The virtual mass of the evaporating droplet}

Consider the system of the drop of diameter $2 \mathrm{~b}$ and density $\rho_{f 1}$ (as shown in Fig. 2) and its nucleating bubble of diameter $2 \mathrm{a}$ and density $\rho_{\mathrm{g} 1}$. Assume that the two spheres are concentric. This configuration was classified by Mori [6] and Oguz and Sadhal [7] as one of the possible configurations encountered in direct-contact evaporation of volatile drops in an immiscible liquid.

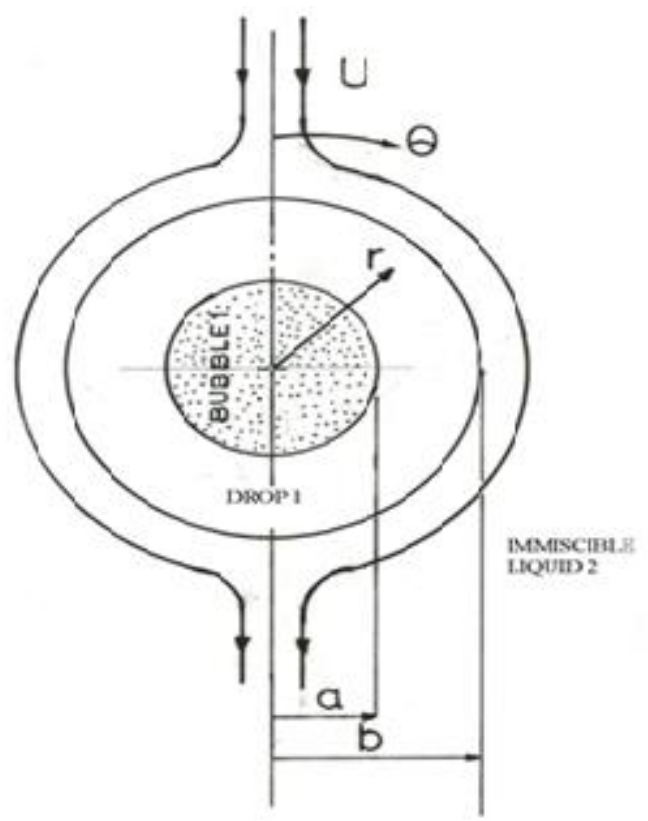

Fig. 2 The geometry of the problem

The irrotational and inviscid flow of the two concentric spheres were given by Lamb [8] and utilized by Kendoush [9]. The total kinetic energy of the fluid surrounding the drop is given by the following (Lamb [8])

$$
K E=\frac{1}{3} \pi \rho_{f 1} \frac{U^{2} a^{3}(1+2 x)}{(1-x)}
$$

where

$$
x=\left(\frac{a}{b}\right)^{3}
$$

Note that the parameter $\mathrm{x}$ can be interpreted as the vaporization ratio as given in Appendix A. If we put $\mathrm{KE}=0.5 \mathrm{MU}^{2}$, then the virtual mass of a drop that contains a small bubble inside it, becomes the following 
$M=\frac{2}{3} \pi \rho_{f 2} \frac{a^{3}(1+2 x)}{(1-x)}$

The virtual mass coefficient is defined according to Cheng, Drew and Lahey [10] by the following:

$\mathrm{C}_{\mathrm{v}}=$ volume of "virtual mass" $\div$ volume of fluid displaced by the evaporating drop

Using Eq. (3), we get the following:

$C_{v}=\frac{(1+2 x)}{2(1-x)}$

This is a new equation that has not been reported before. As $x \rightarrow 0, C_{v}=\frac{1}{2}$ which is the virtual mass coefficient of the single sphere as given by Lamb [8]. This equation is illustrated in Fig. 3.

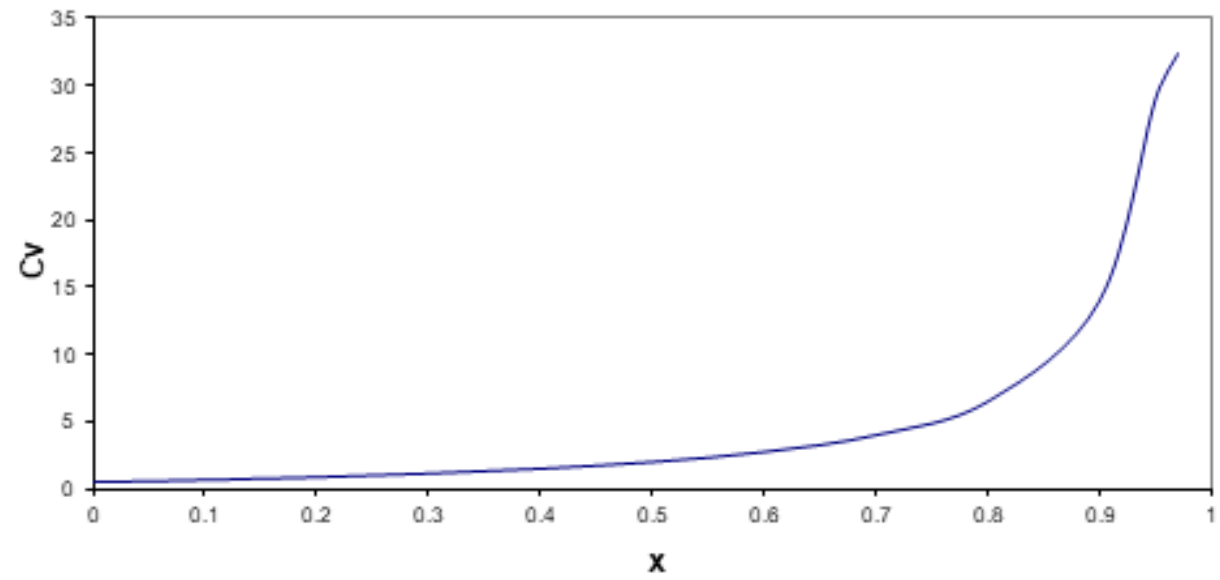

Fig. 3 The relationship between the virtual mass coefficient and the vaporization ration according to Eq. (4).

\subsection{The drag force on the evaporation droplet}

Consider a fixed droplet in a stream of velocity $U$ around it. The tangential shear stress at the surface of two-phase droplet is given as follows:

$$
\tau_{r \theta}=\mu_{2}\left[r \frac{\partial}{\partial r}\left(\frac{u}{r}\right)+\frac{\partial v}{r d \theta}\right]_{r=b}
$$

Substituting Eqs. (B4) and (B5) from Appendix B, into Eq. (5) gives

$$
\tau_{r \theta}=\frac{3 \mu_{2} U \sin \theta}{b(1-x)}
$$

Utilizing the method of integrating the viscous dissipation function (Kendoush [9]), we get 


$$
E=\int_{0}^{\pi}\left[\tau_{r \theta} \nu\right]_{r=b} 2 \pi b^{2} \sin \theta d \theta
$$

Substituting Eq. (6) and Eq. (B5) into Eq. (7) gives

$$
E=8 \pi b \mu_{2} U^{2} \frac{\left(x+\frac{1}{2}\right)}{(1-x)^{2}}
$$

The drag force is given as follows

$$
F=\frac{E}{U}=8 \pi b \mu_{2} U \frac{\left(x+\frac{1}{2}\right)}{(1-x)^{2}}
$$

The drag coefficient is defined by the following

$$
C_{D}=\frac{F}{\frac{1}{2} \rho_{f 2} U^{2}\left(\pi b^{2}\right)}
$$

The substitution of Eq. (9) into Eq. (10) yields:

$$
C_{D}=\frac{32\left(x+\frac{1}{2}\right)}{\operatorname{Re}(1-x)^{2}}
$$

where

$$
\operatorname{Re}=\rho_{f 2} U(2 b) / \mu_{2}
$$

as $x \rightarrow 0$, the above equation reduces to that obtained by Moore [11] for the single spherical bubble. This is also a new equation that has not been reported before, one of the merits of it, is the presence of the parameter $\mathrm{x}$ into it. This makes it useful for providing a fairly accurate balance between buoyancy and drag in the equation of motion.

\subsection{The Equation of Motion}

The droplet gets accelerated due to the continuous evaporation with the increase in size as shown in Fig.1. The unsteady motion of droplet is subjected to the following forces

(i) Buoyancy due to the density difference between the droplet and the continuous phase.

(ii) Buoyancy due to the vapor formation within the droplet

(iii) Drag on the surface of the evaporating droplet

(iv) The impulse due to the virtual mass.

Accordingly, the equation of motion becomes as follows:

$$
\frac{d}{d t}\left(\left[\frac{4}{3} \pi b^{3} \rho_{f 1}+\frac{4}{3} \pi a^{3} \rho_{g 1}+M\right] U\right)=\frac{4}{3} \pi b^{3} g\left(\rho_{f 2}-\rho_{f 1}\right)+\frac{4}{3} \pi a^{3} g\left(\rho_{f 1}-\rho_{g 1}\right)-F
$$


Substituting for M and F from Eqs. (3) and (9) respectively into Eq.(12), we get:

$$
\begin{aligned}
& \frac{4}{3} \pi b^{3} \rho_{f 1} \frac{d U}{d t}+4 \pi \rho_{f 1} U b^{2} \frac{d b}{d t}+\frac{4}{3} \pi a^{3} \rho_{g 1} \frac{d U}{d t}+4 \pi \rho_{g 1} U a^{2} \frac{d a}{d t}+\frac{2}{3} \pi \rho_{f 2} \frac{a^{3}(1+2 x)}{(1-x)} \frac{d U}{d t}+ \\
& 2 \pi \rho_{f 2} a^{2} \frac{(1+2 x) U}{(1-x)} \frac{d b}{d t}+2 \pi \rho_{f 2} \frac{a^{3} U}{(1-x)^{2}}\left(\frac{d x}{d t}\right)=\frac{4}{3} \pi b^{3} g\left(\rho_{f 2}-\rho_{f 1}\right)+\frac{4}{3} \pi a^{3} g\left(\rho_{f 1}-\rho_{g 1}\right) \\
& -8 \pi b \mu_{2} U \frac{\left(x+\frac{1}{2}\right)}{(1-x)^{2}}
\end{aligned}
$$

From Eq. (3), we get the following:

$$
\frac{d x}{d t}=3 b^{-3} a^{2} \frac{d a}{d t}-3 b^{-4} a^{3} \frac{d b}{d t}
$$

Assuming that $\frac{d a}{d t}=\frac{d b}{d t}$ by the same magnitude. This assumption could be proved in a separate study. Consideration should be given to the displacement of the fluid inside the drop. The vapor pressure force aids this force. In opposition to these forces, there are the surface tension force of the drop and the local hydrostatic pressure force of the immiscible liquid. The above assumption, Eq. (14) and Eq. (2) gives the following

$$
\frac{d x}{d t}=\frac{3 x}{b}\left(\frac{b}{a}-1\right) \frac{d a}{d t}
$$

Equation (13) will be solved for two different cases; the first is for low Jakob numbers, that is, Ja $<16$. Riznic et al. [12] recommended the following equation

$$
a=\pi J a\left(\frac{\alpha t}{3}\right)^{\frac{1}{2}}
$$

and

$$
\frac{d a}{d t}=\frac{\pi}{2} J a\left(\frac{\alpha}{3 t}\right)^{\frac{1}{2}}
$$

Substituting Eqs. (15-17) into Eq. (13) and simplifying it yields 


$$
\begin{aligned}
& \frac{d U}{d t}\left(\frac{2 x^{\frac{-1}{3}}}{3}+\frac{2 \rho_{g 1}}{3 x^{\frac{-2}{3}}}+\frac{\rho_{f 2} x^{\frac{2}{3}}(1+2 x)}{3(1-x)}+\frac{U}{t}\left(\rho_{f 1}+\rho_{g 1} x^{\frac{2}{3}}+\frac{\rho_{f 1} x^{\frac{2}{3}}(1+2 x)}{2(1-x)}\right.\right. \\
& \left.+\frac{3 \rho_{f 2} x^{2}\left(x^{\frac{-1}{3}}-1\right)}{(1-x)^{2}}+\frac{\rho_{f 1} x^{\frac{2}{3}}(1+2 x)}{2(1-x)}+\frac{3 \rho_{f 1} x^{2}\left(x^{\frac{-1}{3}}-1\right)}{(1-x)^{2}}\right)=\frac{2 g}{3 x^{\frac{1}{3}}}\left(\rho_{f 2}-\rho_{f 1}\right) \\
& +\frac{2 g\left(\rho_{f 1}-\rho_{g 1}\right)}{3 x^{\frac{-2}{3}}}-\frac{M_{2} U\left(x+\frac{1}{2}\right) x^{\frac{1}{3}}}{\alpha J a(1-x)^{2} t}
\end{aligned}
$$

This equation will be solved for any fixed value of $\mathrm{x}$, namely, 0.3, 0.5 and 0.7. For $\mathrm{x}=0.5$ and utilizing the experimental data reported by Seleki and Gardon [2] for the evaporation of pentane drops in water. The following physical properties of pentane in water were used in Eq. (18) $\rho_{f 1}=3 \mathrm{~kg} / \mathrm{m}^{3}$

$\rho_{f 2}=997.4_{\mathrm{kg} / \mathrm{m}^{3}}, \rho_{g 1}=3 \mathrm{~kg} / \mathrm{m}^{3}, \mu_{2}=3.5 \times 10^{-4} \mathrm{~kg} / \mathrm{ms}, \alpha_{2}=0.00000017 \mathrm{~m}^{2} / \mathrm{s}, \mathrm{Ja}=6.6$. Accordingly, equation (18) becomes as follows

$$
\frac{d U}{d t}+4.2787 \frac{U}{t}=6.9947
$$

This is a first order linear differential equation that can be solved by the integrating factor (I.F.) method as follows

$$
I . F .=\exp \int 4.2787 \frac{d t}{t}=t^{4.2787}
$$

The solution of Eq.(19) is the following

$$
U t^{4.2787}=1.325 t^{5.2787}+C
$$

where $\mathrm{C}$ is the constant of integration. The initial conditions are

$$
\text { at } \mathrm{t}=\text { to } \rightarrow \mathrm{U}=\mathrm{Uo}
$$

Substituting Eq. (22) into Eq. (21) and evaluating C, yields

$$
U=1.325 t+\left(\frac{t_{o}}{t}\right)^{4.2787}\left[U_{o}-1.325 t_{o}\right]
$$

The travel distance of the droplet is obtained from the following

$$
S=S_{o}+\int_{t_{0}}^{t} U d t
$$

Substituting Eq. (23) into Eq. (24) and integrating, yields: 


$$
S=S_{o}+0.6625 t^{2}-\frac{t_{o}^{4.2787}}{t^{3.2787}} \frac{U_{o}}{3.2787}+\frac{1.325 t_{o}^{5.2787}}{3.2787 t^{3.2787}}-1.325 t_{o}{ }^{2}+\frac{t_{o} U_{o}}{3.2787}
$$

where $S_{\mathrm{o}}$ is the position of the droplet at the instant of separation from the nozzle. The value of $U_{\mathrm{o}}$ was taken as $0.2 \mathrm{~m} / \mathrm{s}$ as it was given by various investigators, e.g. Ref. [1]. For this particular case $t_{0}=0.25$ and $S_{0}=0.02 \mathrm{~m}$. These values were obtained from Fig.3 of Selecki and Gradon [2].

Equation (25) was compared with the experimental data of Selecki and Gradon [2] in Fig.4.

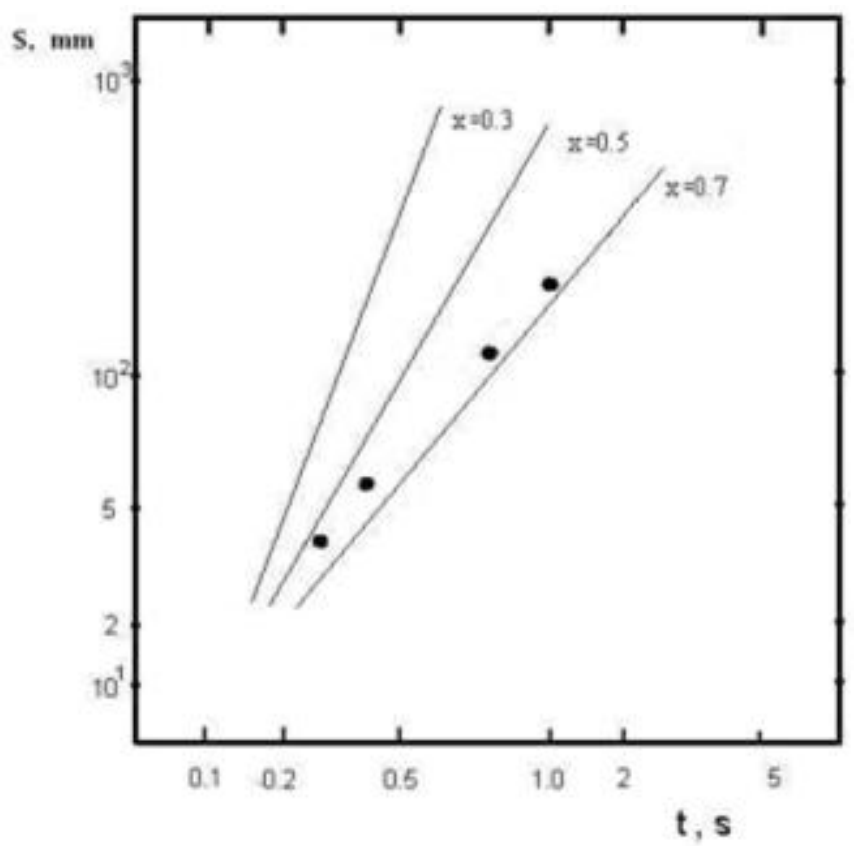

Fig. 4 Comparison with Seleki and Gradon [2], Ja=6.58, $\Delta \mathrm{T}=1.7$ their number 1 in Fig. 3,

Eq. (25).

The calculation was repeated for high Jakob numbers, that is, Ja $>16$ with the following equation that was proposed by Riznic et al. [12]. This is the second case of solving Eq. (13).

$$
a=\pi J a\left(\frac{\alpha t}{3}\right)^{\frac{1}{2}}
$$

and

$$
\frac{d a}{d t}=\frac{\pi}{2} J a\left(\frac{\alpha}{3 t}\right)^{\frac{1}{2}}
$$

Substituting Eqs. (26) and (27) into Eq. (13) with the same previous assumption of $\mathrm{da} / \mathrm{dt}=\mathrm{db} / \mathrm{dt}$, we get the following: 


$$
\begin{aligned}
& \frac{d U}{d t}\left\{\frac{x^{\frac{-1}{3}}}{3}+\frac{\rho_{g 1} x^{\frac{2}{3}}}{3}+\frac{\rho_{f 2}}{6} \frac{x^{\frac{2}{3}}(1+2 x)}{(1-x)}\right\}+\frac{U}{t}\left\{\frac{\rho_{f 1}}{2}+\frac{\rho_{g 1} x^{\frac{2}{3}}}{2}+\frac{\rho_{f 2}}{4} \frac{x^{\frac{2}{3}}(1+2 x)}{(1-x)}+\frac{\rho_{f 2} x\left(x^{\frac{-1}{3}}-1\right)}{(1-x)^{2}}\right\} \\
& =\frac{g}{3} x^{\frac{-1}{3}}\left(\rho_{f 2}-\rho_{f 1}\right)+\frac{g}{3} x^{\frac{2}{3}}\left(\rho_{f 1}-\rho_{g 1}\right)-\frac{18}{\pi} \frac{\mu_{2}}{\alpha J a} \frac{\left(x+\frac{1}{2}\right)}{(1-x)^{2}} x^{\frac{1}{3}}
\end{aligned}
$$

For $\mathrm{x}=0.7, \mathrm{Ja}=50$ and the same physical properties mentioned above, Eq. (28) becomes

$$
\frac{d U}{d t}+8.854 \frac{U}{t}=2.895
$$

The I.F. $=t^{8.854}$ and the solution of Eq. (29) is

$$
U=0.2938 t+\left(\frac{t_{0}}{t}\right)^{8.854}\left[U_{0}-0.2938 t_{0}\right]
$$

The travel distance becomes for the same previous initial conditions

$$
S=0.02083+0.1469 t^{2}-\frac{7.395 \times 10^{-12}}{t^{7.854}}
$$

This equation was compared with the experimental data of Selecki and Gradon [2] in Fig. 5.

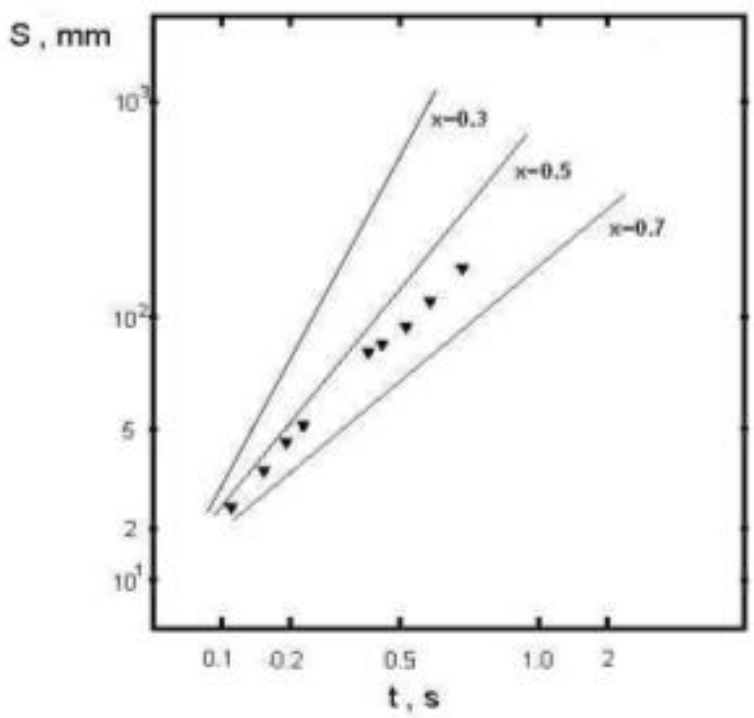

Fig. 5 Comparison with Seleki and Gradon [2] $, \mathrm{Ja}=50, \Delta \mathrm{T}=12.9$ their number 1 in Fig. 3, Eq. (31). 


\section{Discussion}

Figures 4 and 5 show an agreement between the present solution of the equation of motion and the available experimental date of the rise of pentane drop in water for both low and high Ja. Experimental recording of the travel distance of the evaporation drop starts from the nozzle tip, passing through the various stages of evaporation and shape changing and finally into a complete bubble. The agreement between the present solution and the experimental data indicates the validity of the models developed for the virtual mass and the drag.

The present solution may be considered as a quasi steady state solution as it focuses on a particular state of evaporation, e.g. $x=0.5$ but registers the complete history of the droplet from the birth at the nozzle outlet until it reaches the top of the column. The effect of the virtual mass is demonstrated in the present solution and the new model of the drag force seems to produce real and representative solution.

It should be noted that virtual mass coefficient of Eq.(4) is similar to that of Zuber [13] if one replaces $\mathrm{x}$ by the void fraction is bubbly flow. Zuber's formula have been widely mentioned in the literature, e.g. by Wijngaarden [14] and Biesheuvel and Spoelstra [15]. Lamb's [8] derivation of the concentric spheres proved to have dual use. It either represents a cell within a swarm of bubble or it represents a spherical bubble formed within a drop.

A solution of the equation of motion was tried without the inclusion of the virtual mass term (that is, M in Eq. (3) was nullified). The result was an unrealistic solution that gives negative trajectory with time.

In calculating forces on objects in fluids under transient conditions, the history effects, has to be included into the equation of motion. This term is responsible for the diffusion of vorticity around the object, and is, sometimes, called the Basset force (Michaelides [16]). The present analyses did not include this force into it as it was considered to be weak in comparison to the other forces. Murai at al. [17] and Ohl et al. [18] reached the same conclusion.

\section{Conclusions}

Analytical solutions were obtained for the virtual mass coefficient, drag coefficient and the equation of motion of a drop undergoing evaporation while it rises in an immiscible liquid. The following factors were assumed at the droplet:

- transient state

- inviscid and irrotational flow

- incompressible flow

- equal rates of growth for both the bubble and the drop outside it

- the bubble was assumed concentric inside the drop

\section{References}

[1] H.C. Simpson, G.C. Beggs, and M. Nazir, "Evaporation of butane drops in brine," Desalination, vol. 15, pp. 11-23, 1974.

[2] A. Selecki and L. Gradon, "Equation of motion of an expanding vapor drop in an immiscible liquid medium," Int. J. Heat Mass Transfer, vol.19, pp. 925- 929, 1976.

[3] G.K. Raina, R.K. Wanchoo and P.D. Grover, " Direct Contact heat transfer with phase change: Motion of evaporating droplets," AICHE. Journal, vol. 30 (1984) 835-837.

[4] H. Shimaoka and Y.H. Mori, "Evaporation of single liquid: Experiments with n-pentane drops in water and preparation of new heat transfer correlation," Experimental Heat Transfer, vol. 3, pp. 159-172, 1990.

[5] F. Dammel and H. Beer, "Heat transfer from a continuous liquid to an evaporation drop: a numerical analysis," Int. J. Thermal, science, vol. 42, pp. 677-686, 2003.

[6] Y.H. Mori, "Classification of two-phase vapor/liquid bubbles in an immiscible liquid in relation to direct-contact evaporation and condensation processes," Int. J. Multiphase Flow, vol.11, pp. 571-576, 1985.

[7] H.N. Oguz and S.S. Sadhal, "Growth and collapse of translating compound multiphase drops: Analysis of fluid mechanics and heat transfer," J. Fluid Mech., vol. 137, pp. 105-136, 1987.

[8] H. Lamb, Hydrodynamics, The University Press: Cambridge, 6th Ed., p. 124, 1932.

[9] A.A. Kendoush, "Hydrodynamic model for bubbles in a swarm," Chem. Eng. Sci, vol.56 , pp. 235-238, 2001.

[10] L.Y. Cheng, D.A. Drew and R.T. Lahey, Jr., "Virtual mass effect in two- phase flow," NUREG/CR-0020,1978. 
[11] D.W. Moore, "The rise of a gas bubble in a viscous liquid," J. Fluid Mech., vol. 6, pp.161-176, 1959..

[12] J. Riznic, G. Kojasoy and N. Zuber," On the spherically phase change problem," Int. J. Fluid Mech. Research, vol. 26, pp. 110-145, 1999.

[13] N. Zuber, "On the dispersed two-phase flow in the laminar flow regime," Chem. Eng. Sci., vol. 19,pp. 897-917, 1964.

[14] L. van Wijngaarden, "Hydrodynamic interaction between gas bubbles in liquid," J. Fluid Mech., vol. 77, 27-44, 1976.

[15] A. Biesheuvel and S. Spoelstra, "The added mass coefficient of a dispersion of spherical gas bubbles in liquid," Int. J. Multiphase Flow, vol. 15, pp. 911-924,1989.

[16] E.E. Michaelides, "Hydrodynamic force and heat/mass transfer from particles, bubbles and drops- The Freeman Scholar Lecture," J. Fluids Eng., vol.125, pp. 1-30, 2003.

[17] Y. Murai, A. Kitagawa, W-Q. Song, J. Ohta and F. Yamamoto, "Inverse energy cascade structure of turbulence in a bubbles flow (Numerical analysis using Eulerical- Langrangain model equations)," JSME International Journal, vol. 43, pp. 197-205, 2000.

[18] C.D. Ohl, A. Tijink and A. Prosperetti, “The added mass of an expanding bubble," J. Fluid Mech., vol. 482, 271-290, 2003.

[19] A.A. Kendoush, "Theory of convective drop evaporation in direct contact with an immiscible liquid," Desalination, vol.169, pp. 33-41, 2005.

\section{APPENDIX A: The vaporization ratio}

If the vaporization ratio is defined as

$x=1-\frac{m}{m_{o}}$

where $\mathrm{m}$ is the non evaporated mass of liquid within the drop and $\mathrm{m}_{0}$ is the initial mass of the dispersed drop, then

$x=1-\frac{4 / 3 \pi \rho_{3}\left(b^{3}-a^{3}\right)}{4 / 3 \pi \rho_{3} b^{3}}$

or

$\mathrm{x}=(\mathrm{a} / \mathrm{b})^{3}$

\section{APPENDIX B :The velocity field}

The velocity potential of the flow between two concentric spheres, is given by Lamb [8, page 124] as follows

$\phi=\left(A r+\frac{B}{r^{2}}\right) U \cos \theta$

$A=\frac{a^{3}}{b^{3}-a^{3}}$

where $a, b$ are inner and outer sphere radii respectively, and

$B=\frac{1}{2} \frac{a^{3} b^{3}}{b^{3}-a^{3}}$

The velocity components of the flow are obtained as follows 


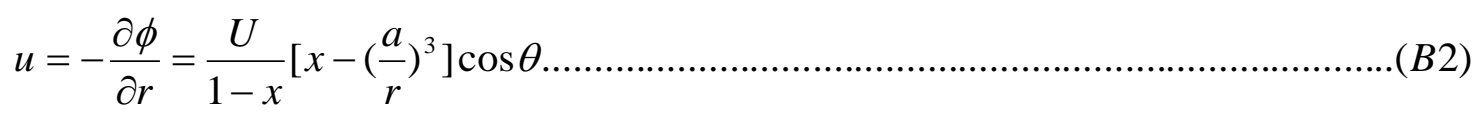

and

$$
v=-\frac{\partial \phi}{r \partial \phi}=\frac{U}{1-x}\left[x+\frac{1}{2}\left(\frac{a}{r}\right)^{3}\right] \sin \theta
$$

The flow field is shown in Fig. 2

The vector $\frac{x U \cos \theta}{1-x}$ in Eq. (B2) and $\frac{x U \sin \theta}{1-x}$ in Eq. (B3) represent the main stream flow around the droplet b. If we want to set the droplet in motion in a stagnant fluid, we simply, remove this vector and we get

$$
u=-\left(\frac{a}{r}\right)^{3} \frac{\cos \theta}{1-x}
$$

and

$$
v=\frac{1}{2}\left(\frac{a}{r}\right)^{3} \frac{\sin \theta}{1-x}
$$

